\title{
ERRATUM
}

\section{The role of $\mathrm{LiO}_{2}$ solubility in $\mathrm{O}_{2}$ reduction in aprotic solvents and its consequences for $\mathrm{Li}-\mathrm{O}_{2}$ batteries}

Lee Johnson, Chunmei Li, Zheng Liu, Yuhui Chen, Stefan A. Freunberger, Praveen C. Ashok, Bavishna B. Praveen, Kishan Dholakia, Jean-Marie Tarascon and Peter G. Bruce

Nature Chemistry 6, 1091-1099 (2014); published online 10 November 2014; corrected after print 20 November 2014.

In the version of this Article originally published, the author list was incorrectly ordered. Jean-Marie Tarascon should have appeared as the penultimate name. This has now been corrected in all online versions of the Article.

\section{CORRIGENDUM}

\section{Discovering chemistry with an ab initio nanoreactor}

Lee-Ping Wang, Alexey Titov, Robert McGibbon, Fang Liu, Vijay S. Pande and Todd J. Martínez

Nature Chemistry 6, 1044-1048 (2014); published online 2 November 2014; corrected after print 20 November 2014.

In the version of this Article originally published, the list of author affiliations was incomplete, and should have appeared as shown below. The list has been corrected in the online versions of the Article.

Lee-Ping Wang ${ }^{1,2}$, Alexey Titov ${ }^{1,2 \dagger}$, Robert McGibbon ${ }^{2}$, Fang Liu ${ }^{1,2}$, Vijay S. Pande ${ }^{2}$ and Todd J. Martínez ${ }^{1,2,3 *}$

${ }^{1}$ The PULSE Institute, Stanford University, Stanford, California 94305, USA. ²Department of Chemistry, Stanford University, Stanford, California 94305, USA. ${ }^{3}$ SLAC National Accelerator Laboratory, Menlo Park, California 94025, USA. ${ }^{\dagger}$ Present address: Advanced Micro Devices, Sunnyvale, California 94088, USA. *e-mail: toddjmartinez@gmail.com 